\title{
Spin wave excitations in the antiferromagnetic state of $\mathrm{Pr}_{0.5} \mathrm{Sr}_{0.5} \mathrm{MnO}_{3}$
}

\author{
V. V. Krishnamurthy ${ }^{\text {a) }}$ \\ Center for Materials for Information Technology, The University of Alabama, Tuscaloosa, Alabama 35487 \\ J. L. Robertson \\ Condensed Matter Sciences Division, Oak Ridge National Laboratory, Oak Ridge, Tennessee 37831 \\ G. J. Mankey \\ Center for Materials for Information Technology, The University of Alabama, Tuscaloosa, Alabama 35487 \\ N. Cavadini and Ch. Niedermayer \\ Laboratory for Neutron Scattering, ETH Zurich and Paul Scherrer Institute, \\ Villigen PSI CH-5232, Switzerland \\ J. F. Mitchell \\ Materials Science Division, Argonne National Laboratory, Argonne, Illinois 60439
}

(Presented on 9 January 2004)

The layered $A$-type antiferromagnet $\mathrm{Pr}_{0.5} \mathrm{Sr}_{0.5} \mathrm{MnO}_{3}$ has been investigated by neutron scattering. Antiferromagnetic ordering is observed with a $T_{N}$ of $152.3 \mathrm{~K}$ and an exponent $\beta$ of $0.148 \pm 0.01$ that suggests $2 d$ Ising behavior. The spin-wave excitations, measured for the neutron momentum transfer $q$ perpendicular to the ferromagnetic planes at $2 \mathrm{~K}$, show a gap of $2.3 \pm 0.2 \mathrm{meV}$ at the zone center $\left[\begin{array}{lll}0.5 & 0 & 0.5\end{array}\right]$. The dispersion near zone center yielded the spin-wave stiffness coefficient $D_{\mathrm{SW}}$ of $\sim 100 \pm 10 \mathrm{meV}^{2}$ which is twice the value found in the isostructural $A$-type antiferromagnet $\mathrm{LaMnO}_{3}$. The double-exchange interaction, introduced by hole doping, enhances $D_{\mathrm{SW}}$ in $\mathrm{Pr}_{0.5} \mathrm{Sr}_{0.5} \mathrm{MnO}_{3}$. The spin wave dispersion at low $q$ fits the dispersion of the Heisenberg model with nearest neighbor exchange interaction and single ion anisotropy. (c) 2004 American Institute of Physics. [DOI: 10.1063/1.1651814]

Doped manganites $\mathrm{R}_{1-x} \mathrm{~A}_{x} \mathrm{MnO}_{3} \quad(\mathrm{R}=$ rare earth, $\mathrm{A}$ $=\mathrm{Ca}^{2+}, \mathrm{Sr}^{2+}$ ) have attracted considerable interest due to their electronic properties such as the large negative magnetoresistance called colossal magnetoresistance (CMR) near a metal-insulator transition, charge ordering, and orbital ordering. ${ }^{1,2}$ In particular, layered manganites exhibit anisotropic magnetic and transport properties. In these compounds, the hole doping at the rare earth site results in mixed valence $(3+$ and $4+)$ for the $\mathrm{Mn}$ ions. The occurrence of metallic ferromagnetism in these mixed valence manganites has been explained within the double-exchange model. ${ }^{3}$ However, it has been shown that the double exchange model is insufficient to explain CMR. ${ }^{4}$ A complete theory of CMR materials is still lacking. It is argued that the CMR in the manganites is associated with the complex interplay of spin and lattice degrees of freedom. The role of lattice polarons has been elucidated both by the theory and the experiments. ${ }^{4,5}$ Recently, several theories of CMR that are based on the electronic phase separation, ${ }^{6}$ the percolation effect, $^{7}$ Griffiths singularity, ${ }^{8}$ and $s$-wave double exchange ${ }^{9}$ have been proposed. The CMR effect is pronounced at the commensurate hole doping concentrations of $1 / 3,1 / 2$, and

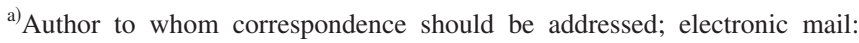
vemuru@bama.ua.edu
}

2/3. ${ }^{10}$ Manganites with such hole concentrations also exhibit charge ordering. ${ }^{11}$ Experiments to probe the fundamental properties of half-doped manganites are necessary to elucidate the relation of magnetic properties to CMR and ordering phenomena.

A few of the half-doped manganites exhibit multiple magnetic phase transitions, such as a transition from an antiferromagnetic ground state to an intermediate ferromagnetic state and to a high temperature paramagnetic state. These half-doped manganites also exhibit CMR at the ferromagnetic to antiferromagnetic transition. ${ }^{10,12}$ The transition has characteristics of a system with complex spin dynamics, such as an exponential relaxation from a usual ferromagnetic or an antiferromagnetic region and a root-exponential relaxation associated with size distributed spin clusters or spin polarons in a glassy region. ${ }^{13}$ Recent investigations by Lorentz electron microscopy show that such clusters have nanoscale $(30-40 \mathrm{~nm})$ dimensions. ${ }^{14}$ It is of current interest to understand this phase transition in order to understand the role played by the nanosized clusters. In the layered $A$-type antiferromagnets, the antiferromagnetism and ferromagnetism are closely connected, i.e., these antiferromagnets are made of alternatively stacked ferromagnetic planes. Therefore, these materials offer an opportunity to study the competition of the ferromagnetic double exchange interaction and the antiferromagnetic super exchange interaction on a charge ordered lattice.
(C) 2004 American Institute of Physics 


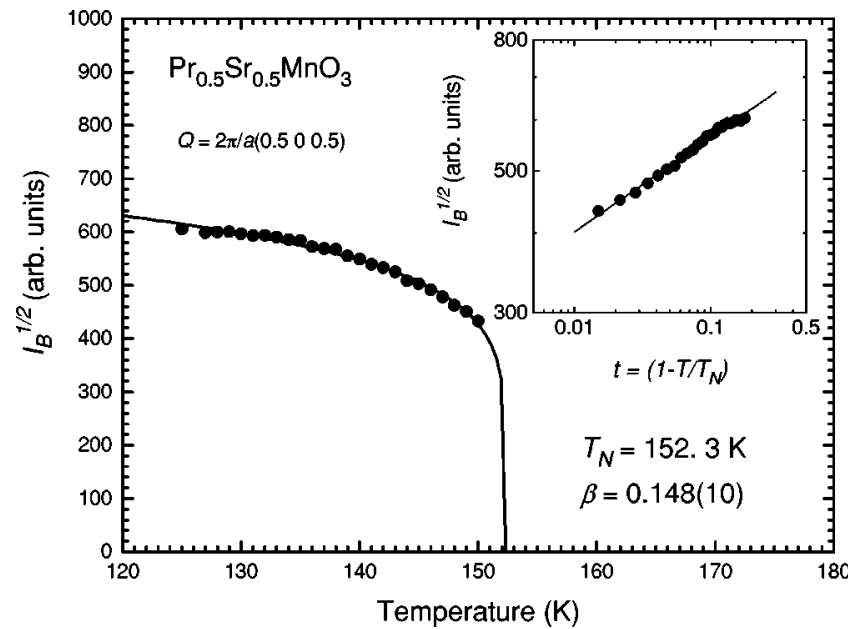

FIG. 1. Temperature dependence of the order parameter at the transition from ferromagnetic antiferromagnetic state derived from the $\left[\begin{array}{lll}0.5 & 0 & 0.5\end{array}\right]$ antiferromagnetic Bragg peak intensity in $\mathrm{Pr}_{0.5} \mathrm{Sr}_{0.5} \mathrm{MnO}_{3}$.

In this study, we focus on the spin dynamics in the layered $A$-type antiferromagnet, the half-doped manganite $\mathrm{Pr}_{0.5} \mathrm{Sr}_{0.5} \mathrm{MnO}_{3}$, which crystallizes in the $P 21 / n$ monoclinic phase. ${ }^{11,15}$ Neutron diffraction studies reported the magnetic moment of $3.1 \mu_{B}$ for $\mathrm{Mn}^{15}$ This compound can be thought of as a mixed valence analogue of the undoped compound $\mathrm{LaMnO}_{3}$ since the later also is an $A$-type antiferromagnet with a $T_{N}$ of $139.5 \mathrm{~K}^{16}$ The spin waves also affect the magnetrotransport. Therefore, an investigation into the nature of spin wave excitations and their relation with the spin and the lattice degrees of freedom is essential for a comprehensive description of magnetism and colossal magnetoresistance.

A single crystal of $\mathrm{Pr}_{0.5} \mathrm{Sr}_{0.5} \mathrm{MnO}_{3}$ was grown by the floating zone method. Magnetic susceptibility measurements show ferromagnetic ordering at $265 \mathrm{~K}$ and a spin reorientation transition to antiferromagnetic state occurs at about 150 $\mathrm{K}$. Elastic and inelastic neutron scattering from the $\mathrm{Pr}_{0.5} \mathrm{Sr}_{0.5} \mathrm{MnO}_{3}$ single crystal was measured at $2 \mathrm{~K}$ with the RITA-2 triple axis spectrometer, which is equipped with a seven blade pyrolytic graphite multicrystal analyzer at SINQ, Paul Scherrer Institute, Switzerland. The instrument was operated at constant momentum transfer with fixed final energy $E_{f}=4.9 \mathrm{meV}$ with a beryllium filter or at $E_{f}=5.6$ or $8 \mathrm{meV}$ with a pyrolytic graphite filter. The sample temperature was controlled between 2 and $190 \mathrm{~K}$ with stability better than 0.1 $\mathrm{K}$ using a ${ }^{4} \mathrm{He}$ cryostat.

Neutron diffraction from the $\left[\begin{array}{lll}0.5 & 0 & 0.5\end{array}\right]$ antiferromagnetic ordering peak was measured as a function of temperature from 2 to $190 \mathrm{~K}$ to investigate the critical behavior of the order parameter at the antiferromagnetic phase transition. The Bragg scattering peak has a linewidth associated with the resolution of the instrument and with the mosaic spread of the crystal domains. The diffraction peak was fitted by a combination of Gaussian functions for quantifying the antiferromagnetic order. Figure 1 shows the square root of the antiferromagnetic $(0.500 .5)$ Bragg peak integrated intensity $I_{B}^{1 / 2}$ as a function of temperature. The intensity decreases as the temperature is increased to approach the transition temperature. $I_{B}^{1 / 2}$, which is proportional to the order parameter,

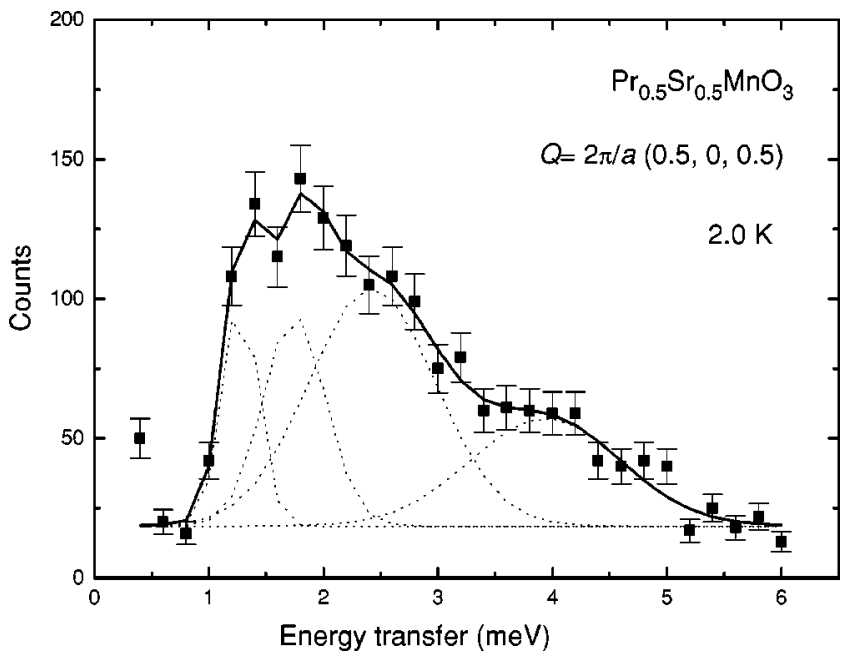

FIG. 2. Neutron scattering intensity as a function of the energy transfer $E$ measured in the antiferromagnetic state at $2 \mathrm{~K}$ for the wave vector transfer $q$ perpendicular to the ferromagnetic planes. The dotted lines show the individual Gaussian functions that are used to model the spectral shape.

i.e., sublattice magnetization $M$, has been fitted by a power law function

$$
l_{B}^{1 / 2} \sim M=M_{0} t^{\beta},
$$

where $M_{0}$ is the sublattice magnetization at $T=0 \mathrm{~K}, t \equiv(1$ $\left.-T / T_{N}\right)$ is the reduced temperature, and $\beta$ is the critical exponent. The inset in Fig. 1 shows $I_{B}^{1 / 2}$ as a function of $t$ in a $\log -\log$ plot. The best fit, which is superimposed over the data in Fig. 1, yielded the Néel temperature $T_{N}=152.3 \mathrm{~K}$ and the critical exponent $\beta=0.148 \pm 0.01$. The value of the Néel temperature is slightly higher than the one reported by Kawano et al. ${ }^{11}$ The critical exponent is close to the Onsager result of exactly $1 / 8$ for the two-dimensional Ising model. ${ }^{17}$

The spin waves were measured by inelastic neutron scattering (INS) at $2 \mathrm{~K}$. INS probes the structure factor $S(Q, \omega)$, which is related to the imaginary part of the susceptibility $\chi^{\prime \prime}(Q, \omega)$ through the fluctuation-dissipation theorem. To extract the information on the structure factor, the inelastic neutron scattering intensity should be convoluted with the instrument resolution function. In the RITA-2 instrument, the seven pyrolytic graphite (002) blades of the analyzer were independently aligned to measure the scattering in the monochromatic point to point focus mode. In this case, the scattered intensity line shape will consist of several Gaussians, with each blade contributing a Gaussian peak to the spectrum. The shape of the spectrum for all the constant $q$ scans could be modeled by a four component Gaussian function, in addition to a constant representing the instrument background. The center of the Gaussian with the highest intensity corresponds to the magnon peak energy. Figure 2 displays a constant $Q$ scan, i.e., the neutron scattering intensity as a function of the energy transfer $E$ measured in the antiferromagnetic state at $2 \mathrm{~K}$ for the wave vector transfer $q$ perpendicular to the ferromagnetic planes. The fit, which is superimposed over the spectrum, yielded a magnon peak energy or the gap of $2.4 \pm 0.2 \mathrm{meV}$. The existence of gap at zone center shows that the dispersion is associated with an optical spin wave branch in which nearest neighbor spins oscillate out of 


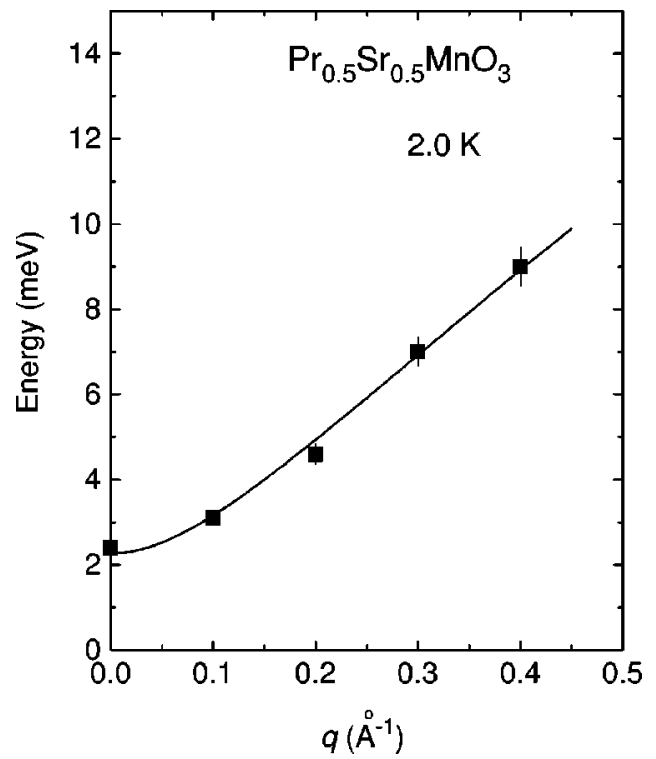

FIG. 3. Spin wave dispersion in the $A$-type antiferromagnet $\operatorname{Pr}_{0.5} \mathrm{Sr}_{0.5} \mathrm{MnO}_{3}$ at $2.0 \mathrm{~K}$. The wave vector transfer $q$ is perpendicular to the ferromagnetic planes.

phase and an antiferromagnetic ground state. Figure 3 displays the wave vector transfer $q$ dependence of the magnon peak energy at the zone center $\left(0.50\right.$ 0.5) in $\operatorname{Pr}_{0.5} \mathrm{Sr}_{0.5} \mathrm{MnO}_{3}$ measured at $2.0 \mathrm{~K}$. This spin wave spectrum at low $q$ has been analyzed with a phenomenological quadratic law, $E$ $=E_{g}+D_{\text {sw }} q^{2}$, where $E_{g}$ is the energy gap and $D_{\text {sw }}$ is the spin-wave stiffness coefficient. The best fit to the data yielded $E_{g}=2.3 \pm 0.2 \mathrm{meV}$ and $D_{\mathrm{sw}}=100 \pm 10 \mathrm{meV} \AA^{2}$. Note that this $D_{\text {sw }}$ value is comparable to $D_{\text {sw }}$ $=117 \mathrm{meV} \AA^{2}$ found in $\mathrm{Nd}_{0.45} \mathrm{Sr}_{0.55} \mathrm{MnO}_{3},{ }^{18}$ and is two times larger than the value of $50 \mathrm{meV}^{2}$ found in $\mathrm{LaMnO}_{3} .{ }^{19}$

The spin-wave spectrum at low $q(\leqslant 0.5)$ could be well described by the dispersion of the Heisenberg Hamiltonian with nearest neighbor exchange interactions and single ion anisotropy. ${ }^{20}$ The best fit displayed as a solid line in Fig. 3 yielded the ferromagnetic exchange integral $J_{1}=0.27 \mathrm{meV}$, the antiferromagnetic exchange integral $J_{2}=-1.83 \mathrm{meV}$, and the single ion anisotropy energy $D=0.057 \mathrm{meV}$. The antiferromagnetic exchange integral is seven times larger than the ferromagnetic exchange integral. This is quite opposite to the case in $\mathrm{LaMnO}_{3}$, where $J_{1}$ is 1.4 times larger than $J_{2}$. Table I presents a comparison of the results of spin-wave dispersion analysis in $\operatorname{Pr}_{0.5} \mathrm{Sr}_{0.5} \mathrm{MnO}_{3}$ with those of the isostructural $A$-type antiferromagnet $\mathrm{LaMnO}_{3}$ reported in Ref. 20. The ferromagnetic coupling $J_{1}$ in $\operatorname{Pr}_{0.5} \mathrm{Sr}_{0.5} \mathrm{MnO}_{3}$ is smaller than the $J_{1}$ in $\mathrm{LaMnO}_{3}$ by a factor of 3. The antiferromagnetic coupling $J_{2}$ in $\operatorname{Pr}_{0.5} \mathrm{Sr}_{0.5} \mathrm{MnO}_{3}$ is larger than the $J_{2}$ in $\mathrm{LaMnO}_{3}$ by a factor of 3 . From the above results, we can infer that in addition to the exchange interactions be-
TABLE I. Comparision of parameters extracted from the spin-wave dispersion measurements in A-type antiferromagnets from inelastic neutron scattering: $S$ is the effective spin of $\mathrm{Mn}$ ions, $J_{1}$ ferromagnetic exchange coupling, $J_{2}$ is the antiferromagnetic exchange coupling, and $D$ is the single ion anisotropy energy.

\begin{tabular}{lcc}
\hline \hline Parameter & $\mathrm{Pr}_{0.5} \mathrm{Sr}_{0.5} \mathrm{MnO}_{3}$ & $\mathrm{LaMnO}_{3}$ \\
\hline$S$ & 1.75 & 2.0 \\
$J_{1}(\mathrm{meV})$ & 0.27 & 0.83 \\
$J_{2}(\mathrm{meV})$ & -1.83 & -0.58 \\
$D(\mathrm{meV})$ & 0.057 & 0.165 \\
\hline \hline
\end{tabular}

tween the spins, the holes also play a role in determining the type of magnetic ordering and the value of Néel temperature in doped manganites.

The research work done at the University of Alabama is supported by the United States Department of Energy through Grant No. DE-FG02-02ER45966 and the National Science Foundation Materials Research Science and Engineering Center program through Grant No. DMR-0213985. This work was also partly supported by Oak Ridge National Laboratory, managed by UT-Battelle, LLC, for the U.S. Department of Energy under Contract No. DE-AC0500OR22725.

${ }^{1}$ Colossal Magnetoresistance, Charge Ordering and Related Properties of Manganese Oxides, edited by C. N. R. Rao and R. Raveau (World Scientific, Singapore, 1998).

${ }^{2}$ Physics of Manganites, edited by T. A. Kaplan and S. D. Mahanti (Kluwer, New York, 1999).

${ }^{3}$ C. Zener, Phys. Rev. 82, 403 (1951).

${ }^{4}$ A. J. Mills, P. B. Littlewood, and B. I. Shraiman, Phys. Rev. Lett. 74, 5144 (1995).

${ }^{5}$ S. J. L. Billinge, R. G. DiFrancesco, G. H. Kwei, J. J. Neumeier, and J. D. Thompson, Phys. Rev. Lett. 77, 715 (1996).

${ }^{6}$ E. Dagatto, T. Hotta, and A. Moreo, Phys. Rep. 344, 1 (2001).

${ }^{7}$ M. Mayr, A. Moreo, J. A. Vergés, J. Arispe, A. Feiguin, and E. Dagotto, Phys. Rev. Lett. 86, 135 (2001).

${ }^{8}$ M. B. Salmon, L. Pin, and S. H. Chun, Phys. Rev. Lett. 88, 197203 (2002).

${ }^{9}$ J. A. Vergés, V. Martín-Mayor, and L. Brey, Phys. Rev. Lett. 88, 136401 (2002).

${ }^{10}$ P. Schiffer, A. P. Ramirez, W. Bao, and S.-W. Cheong, Phys. Rev. Lett. 75, 3336 (1995)

${ }^{11}$ H. Kawano, R. Kajimoto, H. Yoshizawa, Y. Tomiaoka, H. Kuwahara, and Y. Tokura, Phys. Rev. Lett. 78, 4253 (1997).

${ }^{12}$ H. Kuwahara, Y. Tomioka, A. Asamitsu, Y. Moritomo, and Y. Tokura, Science 270, 961 (1995).

${ }^{13}$ V. V. Krishnamurthy, I. Watanabe, K. Nagamine, H. Kuwahara, and Y. Tokura, Phys. Rev. B 61, 4060 (2000).

${ }^{14}$ T. Asaka, Y. Anan, T. Nagai, S. Tsutsumi, H. Kuwahara, K. Kimoto, Y. Tokura, and Y. Matsui, Phys. Rev. Lett. 89, 207203 (2002).

${ }^{15}$ Y. Tomioka, A. Asamitsu, Y. Moritomo, H. Kuwahara, and Y. Tokura, Phys. Rev. Lett. 74, 5108 (1995).

${ }^{16}$ F. Moussa, M. Hennion, J. Rodriguez-Carvajal, H. Moudden, L. Pinsard, and A. Revcolevschi, Phys. Rev. B 54, 15149 (1996).

${ }^{17}$ L. Onsager, Phys. Rev. 65, 117 (1944).

${ }^{18}$ H. Yoshizawa, H. Kawano, J. A. Fernandez-Baca, H. Kuwahara, and Y. Tokura, Phys. Rev. B 58, R571 (1998).

${ }^{19}$ K. Hirota et al., J. Phys. Soc. Jpn. 65, 3736 (1996).

${ }^{20}$ F. Moussa, M. Hennion, G. Biotteau, Rodriguez-Carvajal, L. Pinsard, and A. Revcolevschi, Phys. Rev. B 60, 12299 (1999). 